\title{
Laparoscopic subtotal colectomy for synchronous triple colorectal cancer: A case report
}

\author{
XUETONG JIANG, CHUANQI XU, DONG TANG and DAORONG WANG \\ Department of Gastrointestinal Surgery, Clinical Medical College of Yangzhou University, \\ Subei People's Hospital of Jiangsu, Yangzhou, Jiangsu 225001, P.R. China
}

Received April 4, 2015; Accepted May 23, 2016

DOI: $10.3892 / \mathrm{ol} .2016 .4803$

\begin{abstract}
Synchronous colorectal cancers refer to the simultaneous occurrence of multiple colorectal tumors in a single patient, excluding any metastases from other organs. At present, radical surgery is considered the standard curative treatment; however, individualized surgical strategies depend on tumor location, the depth of invasion and the general health of the patient. In the present study, the case of a 52-year-old man who presented with a 2-month history of abdominal pain that was accompanied by intermittent hematochezia and weight loss is reported. The patient had no family history of cancer. Computed tomography (CT) of the abdomen revealed intestinal wall thickness in the transverse colon and volvulus in the hepatic flexure of colon. Colonoscopy identified 3 tumors: The first tumor was located in the descending colon with lumen stenosis $\sim 60 \mathrm{~cm}$ from the anal verge, the second tumor was located in the hepatic flexure of the colon, and the third tumor was located in the sigmoid colon, $23 \mathrm{~cm}$ from the anal verge. Subsequently, laparoscopic subtotal colectomy was performed and all three tumors were removed, and the diagnosis was confirmed by histopathological examination. The patient did not undergo chemotherapy following surgery, due to personal reasons. Subsequent to 19 months of follow-up examinations using CT and colonoscopy every 6 months, the patient exhibited no signs of recurrence. Thus, laparoscopic subtotal colectomy represents an effective surgical approach for the treatment of synchronous colorectal cancer following imaging and endoscopic diagnosis.
\end{abstract}

Correspondence to: Professor Daorong Wang, Department of Gastrointestinal Surgery, Clinical Medical College of Yangzhou University, Subei People's Hospital of Jiangsu, 98 Nantong West Road, Yangzhou, Jiangsu 225001, P.R. China

E-mail: daorong666@sina.com

Key words: synchronous colorectal cancer, subtotal colectomy, laparoscope

\section{Introduction}

Synchronous cancers are characterized by the simultaneous occurrence of multiple primary tumors in the same patient. Synchronous malignancies most commonly occur in the colon, with a particularly high prevalence in elderly patients $(1,2)$. The occurrence of synchronous colorectal cancers is extremely rare and may be identified at any location within the large intestine (3). Synchronous cancers are relatively uncommon, and triple synchronous colon cancers are particularly rare. The majority of studies have identified that synchronous colorectal cancers more frequently occur in the right colon when compared with single tumors $(4,5)$.

Surgical resection is the primary treatment option for synchronous colorectal cancers. Recently, laparoscopic surgery has been used in synchronous colorectal cancers successfully, as evaluated by certain studies; however, controversy remains concerning operative procedures for multiple segmental resections, and total or subtotal colectomy $(6,7)$. Thus, at present, no standard treatment for synchronous colon cancer has been established. There is no difference in survival between synchronous colorectal cancers and single colorectal cancers, if resections are curative. In addition, the pathological stages between these two types of tumor are identical $(5,8,9)$. The present study reports the case of a 52-year-old male patient who presented with triple synchronous cancer arising from the colon, which was successfully treated with laparoscopic subtotal colectomy.

\section{Case report}

A 52-year-old man was admitted to Subei People's Hospital of Jiangsu (Yangzhou, China) on October 3, 2014, and presented with a 2-month history of abdominal pain, intermittent hematochezia and weight loss. The patient had no significant medical history, no family history of cancer and was a non-smoker. Physical examination revealed deep tenderness at the left lower quadrant of the abdomen without rebound tenderness on palpation. Laboratory examinations revealed no significant abnormal results. Hemoglobin and cancer tumor markers, including cancer antigen 19-9, $\alpha$-fetoprotein, carcinoembryonic antigen and prostate-specific antigen were all within the normal ranges. In addition, chest X-ray, electrocardiogram, ultrasonic cardiogram and pulmonary function tests were all 


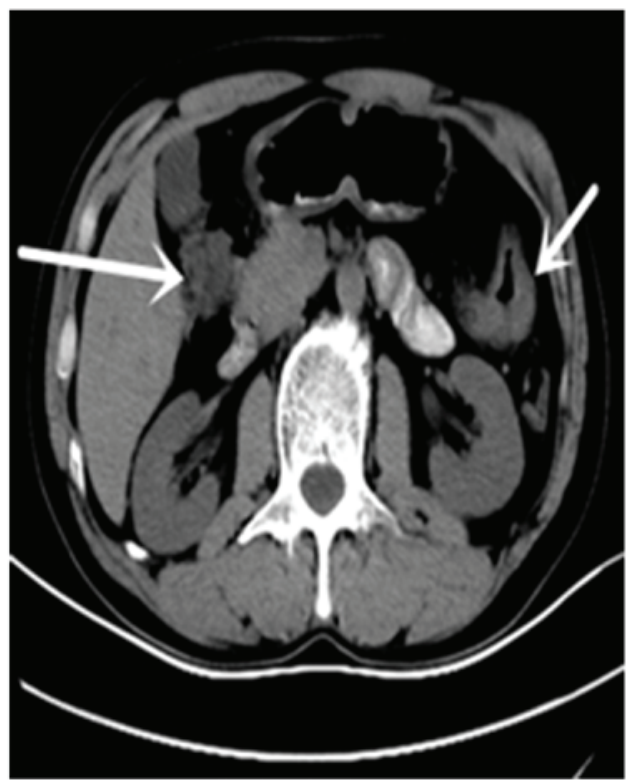

Figure 1. Abdominal computed tomography scan revealing a tumor of hepatic flexure of the colon (left arrow). A tumor of the descending colon is also visible (right arrow)

normal. Computed tomography (CT) of the abdomen revealed marked intestinal wall thickness in the transverse colon and volvulus in the hepatic flexure of the colon (Fig. 1). Colon cancer was suspected.

Colonoscopy identified 3 tumors: The first tumor was located in the descending colon, with lumen stenosis observed $\sim 60 \mathrm{~cm}$ from the anal verge; the second tumor was located in the hepatic flexure of colon; and the third tumor was located in the sigmoid colon, $23 \mathrm{~cm}$ from the anal verge (Fig. 2A). Histopathological examination of biopsy specimens led to a diagnosis of adenocarcinoma for the tumors of the descending colon and the hepatic flexure of the colon, while the sigmoid tumor was identified as tubulovillous adenoma with moderate epithelial dysplasia. Biopsy specimens were fixed in formalin, embedded in paraffin, sliced to a $5 \mu \mathrm{m}$-thickness and stained with hematoxylin and eosin. The results of staining revealed atypical cells that were adenoid with papillary or villous distribution and invasive growth. Synchronous adenocarcinoma of the descending colon and the hepatic flexure of colon was confirmed by colonoscopy and pathological examination (Fig. 2B).

Subsequent laparoscopic exploration confirmed the presence of one tumor (diameter, $6 \mathrm{~cm}$ ) in the hepatic flexure of the colon, a second tumor (diameter, $5 \mathrm{~cm}$ ) in the descending colon and a third tumor (diameter, $2 \mathrm{~cm}$ ) in the sigmoid colon, without serous invasion. No metastatic tumors were identified in the abdominal cavity or liver. However, exploration revealed enlarged lymph nodes in the mesentery proximal to the hepatic flexure of the colon and descending colon. Subsequently, an extended hemicolectomy from the terminal ileum to the sigmoid was performed under laparoscopy (Fig. 3). The right half of the colon was separated, followed by the left half, and end-to-side anastomosis between the jejunum and sigmoid colon was performed. The operative duration was $160 \mathrm{~min}$ with intraoperative blood loss of $\sim 100 \mathrm{ml}$.

Resected tissues were were fixed in formalin, embedded in paraffin, sliced to a $5 \mu \mathrm{m}$-thickness and stained with
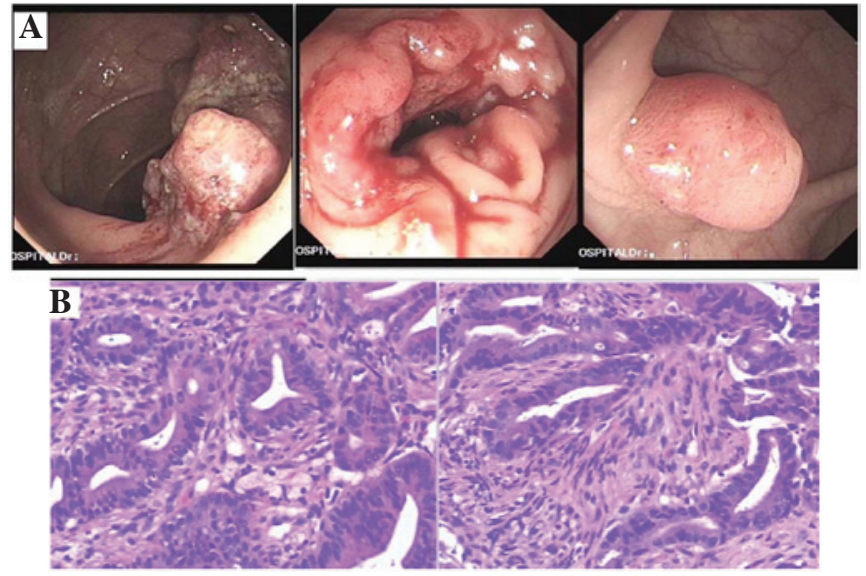

Figure 2. (A) Colonoscopy images showing 3 tumors. One cauliflower-like tumor with lumen stenosis is located at the descending colon (middle panel). Another cauliflower-like tumor is located at the hepatic flexure of colon (left panel). The third adenomatoid polyp tumor $\sim 1.5 \times 1.5 \mathrm{~cm}$ is located at the sigmoid colon $23 \mathrm{~cm}$ from the anal verge (right panel). (B) Histopathological analysis of the biopsy specimen revealed atypical cells that were adenoid with papillary or villous distribution, with invasive growth. Hematoxylin and eosin staining; magnification, x200.

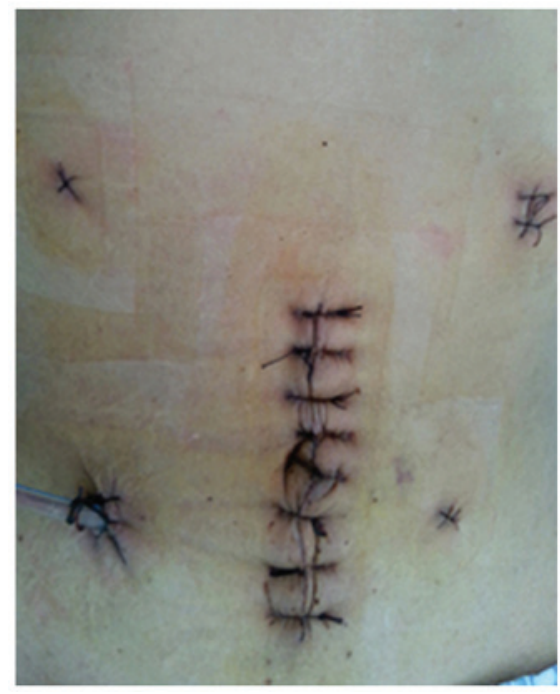

Figure 3. Surgical incisions were required for laparoscopic subtotal colectomy.

hematoxylin and eosin. The results of staining revealed that the cancerous cells were arranged in a tubular arrangement, and cells had large nuclei with deep staining, with lighter staining of the cytoplasm. Staining also revealed invasive growth. Histopathological examination of the resected tissue specimens revealed 3 tumors (Fig. 4). The tumor at the hepatic flexure of the colon was identified as protruding adenocarcinoma and a foci with mucinous adenocarcinoma, which had invaded up to the serous membrane. Another tumor was observed at the descending colon, which was infiltrating adenocarcinoma, which had also invaded up to the serous membrane. The third tumor was located in the sigmoid colon $\sim 3.5 \mathrm{~cm}$ from the sigmoid valve, which was diagnosed as adenocarcinoma with invasion of the submucosa. In addition, 26 regional lymph nodes without any metastasis were resected; however, hematoxylin and eosin staining and immunohistochemical examination of the 


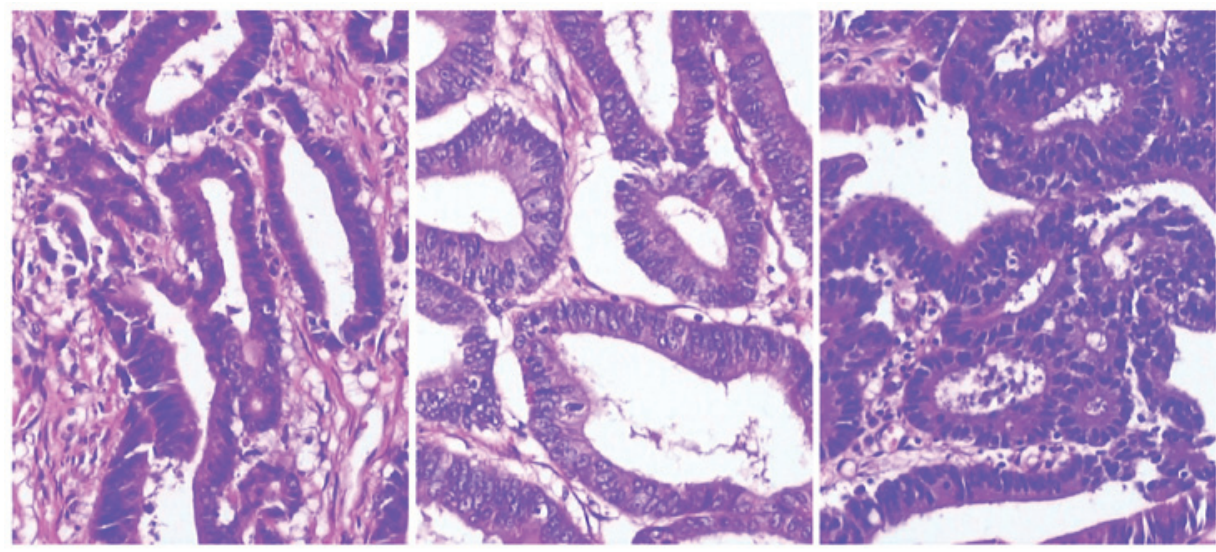

Figure 4. Histopathological examination of the removed tissue specimens revealed 3 tumors, which exhibited cancerous cells arranged in a tubular pattern and invasive growth with large cell nuclei with deep staining, and lighter staining in the cytoplasm. Magnification, x200.

appendix indicated tumor invasion. Laparoscopic surgery was considered superior to traditional open surgery as it causes less trauma, resulting in a quick recovery. The patient recovered well and was discharged 10 days after surgery. The patient did not undergo chemotherapy following surgery, due to personal reasons. Subsequent to 19 months of follow-up examinations, which consisted of CT and colonoscopy every 6 months, the patient exhibited no signs of recurrence. The present study was approved by the Ethical Committee of Subei People's Hospital of Jiangsu and written informed consent was obtained from the patient.

\section{Discussion}

Synchronous colorectal cancers are defined as tumors that are diagnosed at the same time as or within 6 months of the initial diagnosis of another primary tumor, excluding metastatic lesions from other primary cancers. Generally, synchronous tumors occur $\geq 4 \mathrm{~cm}$ from each other $(10,11)$. With the development of diagnostic technologies, increasing numbers of synchronous colorectal cancers are identified clinically. The prevalence of synchronous colorectal carcinoma has been reported to range between 3 and 6\% (10-13). However, the actual incidence is considered to be higher as not all tumors are identified by colonoscopy. A number of tumors may remain undetected due to bowel obstruction or poor bowel preparation (2). Furthermore, certain tumors at early histological stages cannot be verified by histopathological examination of colonoscopy (14). In the present study, a rare case of triple synchronous cancer arising from the hepatic flexure of the colon, descending colon and the sigmoid in a 52-year-old male was reported. Notably, the patient had no family history of cancer or genetic predisposing factors.

The preoperative or intraoperative diagnosis of multiple synchronous colorectal carcinomas is extremely important, but remains challenging. It is difficult to identify certain small tumors on CT, and sometimes complete examination of the large intestine cannot be conducted, due to intestinal lumen stenosis. The combined use of CT and colonoscopy has been reported as a useful tool for the preoperative evaluation of synchronous colorectal carcinoma $(15,16)$. Colonoscopic examination may provide clear images of the whole colon.
CT is also a sensitive examination for synchronous colorectal carcinoma, particularly in patients that have undergone incomplete colonoscopy (17); however, a number of small tumors may be missed. In the present case, 2 tumors were identified by CT, while colonoscopy revealed 3 tumors. (Fig. 2A). It was initially unclear whether the lesion in the sigmoid colon was a benign or malignant tumor. Additional imaging examinations, such as magnetic resonance colonography and positron emission tomography-CT have also been reported as useful tools for the diagnosis of synchronous colorectal carcinoma $(18,19)$.

Surgical resection is the most common treatment for synchronous colorectal cancers. However, initial surgical procedures for multiple colorectal carcinomas remain controversial; surgical procedures for multiple lesions or cancers should be individualized according to the tumor location, depth of invasion and the general health of the patient. Previous studies have recommended the use of total or subtotal colectomy to resect any potential existing synchronous tumors or polyps that have not been detected in patients exhibiting lesions in separate segments (20-23). However, another study reported that traditional surgical methods should be used for multiple segmental resections to preserve the normal colon (24). In patients with tumors in adjacent segments, a more extensive resection, including removal of the proximal intestinal and local lymph nodes, is required (5). Radical curative surgery for synchronous triple colorectal cancer aims to remove all tumors. Certain studies have recommended that intraoperative colonoscopy should be conducted to confirm that no additional tumors are present $(2,25)$. However, the effectiveness of intraoperative colonoscopy is controversial as the procedure increases the risk of infection (26). In the present case, an extended hemicolectomy from the terminal ileum to sigmoid was performed under laparoscopy with ileosigmoidostomy anastomosis, and thus we hypothesized that subtotal colectomy would ensure that all tumors were removed. Histopathological examination of the resected tissue specimens confirmed that all tumors had been resected.

A previous study indicated that subtotal colectomy may increase defecation frequency as the normal colon cannot be preserved (27). In the present case, the patient exhibited normal defecation by the third postoperative day without diarrhea. Thus, in the present case laparoscopic subtotal 
colectomy was successful in removing all 3 tumors without any serious side effects.

In summary, the present study reported a case of triple synchronous colorectal cancer in a patient with no family history of cancer. The first tumor was located in the descending colon with lumen stenosis, $\sim 60 \mathrm{~cm}$ from the anal verge, the second tumor was located in the hepatic flexure of colon and the third lesion was located in the sigmoid colon, $23 \mathrm{~cm}$ from the anal verge. All three tumors were removed by laparoscopic subtotal colectomy. Therefore, laparoscopic subtotal colectomy presents an effective surgical approach for synchronous colorectal cancer following imaging and endoscopic diagnosis.

\section{Acknowledgements}

This study was supported by The Department of Gastrointestinal Surgery of Subei People's Hospital.

\section{References}

1. Fukatsu H, Kato J, Nasu JI, Kawamoto H, Okada H, Yamamoto H, Sakaguchi K and Shiratori Y: Clinical characteristics of synchronous colorectal cancer are different according to tumour location. Dig Liver Dis 39: 40-46, 2007.

2. Yeh CC, Hsi SC, Chuu CP and Kao YH: Synchronous triple carcinoma of the colon and rectum. World J Surg Oncol 11: 66, 2013.

3. Yang J, Peng JY and Chen W: Synchronous colorectal cancers: A review of clinical features, diagnosis, treatment, and prognosis Dig Surg 28: 379-385, 2011.

4. Chen HS and Sheen-Chen SM: Synchronous and "early" metachronous colorectal adenocarcinoma: Analysis of prognosis and current trends. Dis Colon Rectum 43: 1093-1099, 2000.

5. Passman MA, Pommier RF and Vetto JT: Synchronous colon primaries have the same prognosis as solitary colon cancers. Dis Colon Rectum 39: 329-334, 1996.

6. Hirano Y, Hattori M, Sato Y, Maeda K, Douden K and Hashizume Y: Concurrent single-incision laparoscopic right hemicolectomy and sigmoidectomy for synchronous carcinoma: Report of a case. Indian J Surg 75: 293-295, 2013.

7. Bardakcioglu O and Ahmed S: Single incision laparoscopic total abdominal colectomy with ileorectal anastomosis for synchronous colon cancer. Tech Coloproctol 14: 257-261, 2010.

8. Latournerie M, Jooste V, Cottet V, Lepage C, Faivre J and Bouvier AM: Epidemiology and prognosis of synchronous colorectal cancers. Br J Surg 95: 1528-1533, 2008.

9. Nikoloudis N, Saliangas K, Economou A, Andreadis E, Siminou S, Manna I, Georgakis K and Chrissidis T: Synchronous colorectal cancer. Tech Coloproctol 8 Suppl 1: s177-179, 2004.
10. Cunliffe WJ, Hasleton PS, Tweedle DE and Schofield PF: Incidence of synchronous and metachronous colorectal carcinoma. Br J Surg 71: 941-943, 1984.

11. Mulder SA, Kranse R, Damhuis RA, de Wilt JH, Ouwendijk RJ, Kuipers EJ and van Leerdam ME: Prevalence and prognosis of synchronous colorectal cancer: A Dutch population-based study. Cancer Epidemiol 35: 442-447, 2011.

12. Welch JP: Multiple colorectal tumors. An appraisal of natural history and therapeutic options. Am J Surg 142: 274-280, 1981.

13. Derwinger K and Gustavsson B: A study of aspects on gender and prognosis in synchronous colorectal cancer. Clin Med Insights Oncol 5: 259-264, 2011.

14. Hancock RJ: Synchronous carcinoma of the colon and rectum. Am Surg 41: 560-563, 1975.

15. Kim MS and Park YJ: Detection and treatment of synchronous lesions in colorectal cancer: The clinical implication of perioperative colonoscopy. World J Gastroenterol 13: 4108-4111, 2007.

16. McArthur DR, Mehrzad H, Patel R, Dadds J, Pallan A, Karandikar SS and Roy-Choudhury S: CT colonography for synchronous colorectal lesions in patients with colorectal cancer: Initial experience. Eur Radiol 20: 621-629, 2010.

17. Pang EJ, Liu WJ, Peng JY, Chen NW and Deng JH: Prediction of synchronous colorectal cancers by computed tomography in subjects receiving an incomplete colonoscopy: A single-center study. World J Gastroenterol 21: 1857-1864, 2015.

18. Achiam MP, Holst Andersen LP, Klein M, Chabanova E, Thomsen HS and Rosenberg J: Preoperative evaluation of synchronous colorectal cancer using MR colonography. Acad Radiol 16: 790-797, 2009.

19. Kinner S, Antoch G, Bockisch A and Veit-Haibach P: Whole-body PET/CT-colonography: A possible new concept for colorectal cancer staging. Abdom Imaging 32: 606-612, 2007.

20. Wang HZ, Huang XF, Wang Y, Ji JF and Gu J: Clinical features, diagnosis, treatment and prognosis of multiple primary colorectal carcinoma. World J Gastroenterol 10: 2136-2139, 2004.

21. Slater G, Aufses AH Jr and Szporn A: Synchronous carcinoma of the colon and rectum. Surg Gynecol Obstet 171: 283-287, 1990.

22. Easson AM, Cotterchio M, Crosby JA, Sutherland H, Dale D, Aronson M, Holowaty E and Gallinger S: A population-based study of the extent of surgical resection of potentially curable colon cancer. Ann Surg Oncol 9: 380-387, 2002.

23. Enker WE and Dragacevic S: Multiple carcinomas of the large bowel: A natural experiment in etiology and pathogenesis. Ann Surg 187: 8-11, 1978.

24. Adloff M, Arnaud JP, Bergamaschi R and Schloegel M: Synchronous carcinoma of the colon and rectum: Prognostic and therapeutic implications. Am J Surg 157: 299-302, 1989.

25. Kaibara N, Kimura O, Nishidoi H, Miyano Y and Koga S: Intraoperative colonoscopy for the diagnosis of multiple cancers of the large intestine. Jpn J Surg 12: 117-121, 1982.

26. Torralba JA, Robles R, Parrilla P, Lujan JA, Liron R, Piñero A and Fernandez JA: Subtotal colectomy vs. intraoperative colonic irrigation in the management of obstructed left colon carcinoma. Dis Colon Rectum 41: 18-22, 1998.

27. Holubar SD, Wolff BG, Poola VP and Soop M: Multiple synchronous colonic anastomoses: Are they safe? Colorectal Dis 12: $135-140,2010$. 